\title{
Optical Method to Study Post-Necking Material Behaviour
}

\author{
M. V. Erpalov a) and V. A. Khotinov \\ Ural Federal University named after the first President of Russia B.N. Yeltsin, 620002 Ekaterinburg, Russia \\ ${ }^{\text {a)} C o r r e s p o n d i n g ~ a u t h o r: ~ m . v . e r p a l o v @ u r f u . r u ~}$
}

\begin{abstract}
The article is devoted to the determination of equivalent stresses and strains in the minimum cross-section of the neck during tensile testing of cylindrical specimens. To correct the average tensile stresses, the Bridgman theory is used, based on the experimental measurement of the neck. The paper proposes an approach for calculating the radius of curvature of the neck based on the approximation equation that describes the entire profile of specimen. Its accuracy was estimated using the finite element method. A program has been developed that provides the measure of the diameter of specimen in the minimum cross-section of the neck and the calculation of the radius of its curvature from the video recording of the test process based on the proposed equation. Using this program, hardening curves for 09G2S steel were constructed at engineering, true and equivalent stresses.
\end{abstract}

\section{INTRODUCTION}

Tensile testing of specimens is the most common approach to study the mechanical properties of metals and alloys, and it is one of the main acceptance tests for metallurgical products. However, the study of the reliable properties of materials after localization of deformation in the neck is very rare. There are two main reasons for this. Firstly, the existing standards for metallurgical products limit the field of studying mechanical properties up to tensile strength; therefore, for engineering practice, the determination of equivalent stresses and strains in the neck is not of great importance. On the other hand, for researchers and scientists, the neck formation is of particular interest: on a basis of equivalent stress and strain, you can define the hardening curve of a material, calibrate different fracture models, study the effects of high strain rates, temperature or stress triaxiality, etc. However, researchers commonly face inextricable difficulties associated with the need to take into account the inhomogeneous stress distribution in the neck of the specimen.

According to Bridgman [1], the stress in the neck can be determined using three degrees of approximation. The first one does not take into account the presence of a neck at all. To calculate stress in this case you should divide the tensile load $(P)$ by the area of the original cross-section of specimen $\left(A_{0}\right)$. This approach is the basis for engineering practice. The second approximation takes into account the localization of deformation and deals with the average values of stress in the neck. In this case, the stress is calculated by dividing the tensile load by the current value of the cross-sectional area $(A)$. This approach has a closer correlation with the reliable properties of a material; however, the average stress is unsuitable for describing many physical phenomena including fracture. In this case, a third approximation is necessary, taking into account the inhomogeneous distribution of stresses in the axial and radial directions. As Bridgman showed, as well as a number of other researchers, [2], stress inhomogeneity can be taken into account by experimentally measuring the neck profile, namely, the diameter in the minimum crosssection $(d)$ and the radius of neck curvature $(R)$.

This work aims to develop a tool, based on the video recording of the test process, which will provide experimentally measuring the neck profile at any instant up to specimen failure. When developing such a tool, the main task we set was the ability to quickly and accurately process experimental data. Therefore, we propose an analytical description of the neck profile, which on the one hand takes into account the difference in the properties of materials, and on the other hand allows the use of mathematical tools to calculate the radius of neck curvature, not the measurement. The speed and ease of processing experimental data is based on the use of Wolfram Language.

High-Energy Processes in Condensed Matter (HEPCM 2020)

AIP Conf. Proc. 2288, 030007-1-030007-7; https://doi.org/10.1063/5.0028748

Published by AIP Publishing. 978-0-7354-4018-0/\$30.00 


\section{METHODOLOGY}

\section{The Analytical Description of Neck Profile}

The current value of the radius of neck curvature is the only parameter that allows you to take into account the inhomogeneous distribution of stresses in the minimum cross-section of specimen. To determine its values, projectors [1] or instrumental microscopes [3] are used. However, in this case, the measurement of the radius of curvature is subjective and requires the specimen being removed from the test setup. Nowadays, the method of digital image correlation (DIC) is widely used [4, 5]. Nevertheless, its scope is very limited without the use of an additional camera: using DIC, the deformation is determined on the surface of mainly flat specimens, and the obtained data are used to solve the inverse problem by the finite element method (FEM). The most accurate methods for measuring the neck of specimens were obtained using the DSLR and high-speed cameras in works of Sancho et al. [6, 7] and Mirone et al. [8, 9]. However, Sancho does not reveals the method to calculate the radius of neck curvature. Mirone, on the other hand, uses the fitting techniques to approximate the profiles by way of second or third order polynomials and then calculates derivatives of the polynomials and finally desired curvature values. However, he notes that this procedure is very time-consuming and may induce errors up to more than $10 \%$. Assuming that the change in neck profile is independent of material properties, he proposes a regression model called MLR for characterization of post-necking material behaviour. However, this approach requires strong experimental evidence for a wide range of materials.

Based on the mathematical analysis of the FEM results performed in [10], in this paper, we propose to approximate the neck profile using the equation:

$$
\rho=\frac{d_{1}}{2}-\frac{d_{1}-d}{2} \cdot\left(1+\frac{z^{2}}{c}\right)^{-1},
$$

where $\rho$ and $z$ are radial and axial coordinates of points on specimen surface respectively, $c$ is a parameter sensitive to a material properties, $d$ is a minimum neck diameter and $d_{1}$ is a diameter of specimen at the beginning of necking.

Equation 1 suggests that the neck profile is symmetrical about a plane perpendicular to the axis of specimen and passing through the minimum cross-section of neck. It allows you to calculate the radius of neck curvature quite simply:

$$
R=\frac{c}{d_{1}-d}
$$

The results of additional computer simulation of tensile tests, performed in this work, show that the proposed analytical description of the neck profile is effective regardless of the nature of the material hardening curve and the instant of the test (Fig. 1). The largest error in the analytical description of the neck was equal to $1.09 \%$ for the data presented in Fig. 1 (a), and $0.73 \%$ - in Fig. 1 (b). For a better approximation of the neck profile, we recommend to use the fitting techniques, taking into account the variation of all parameters included in Eq. 1 despite their physical concept. 


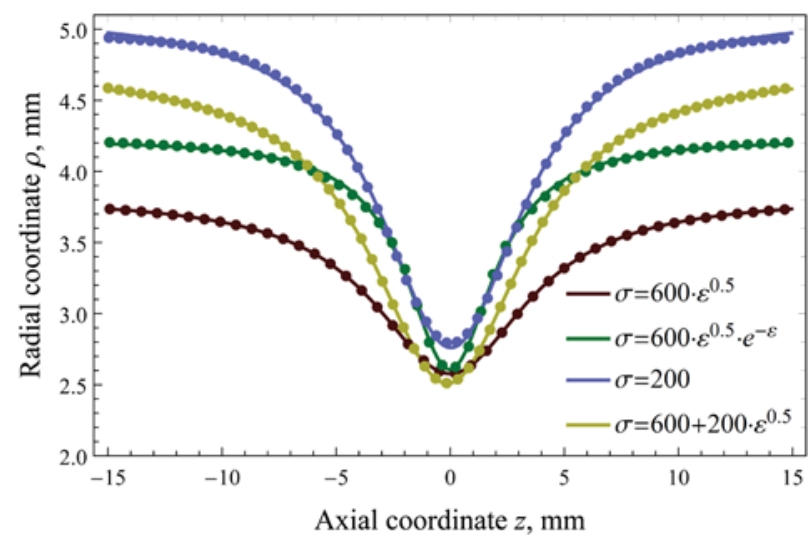

(a)

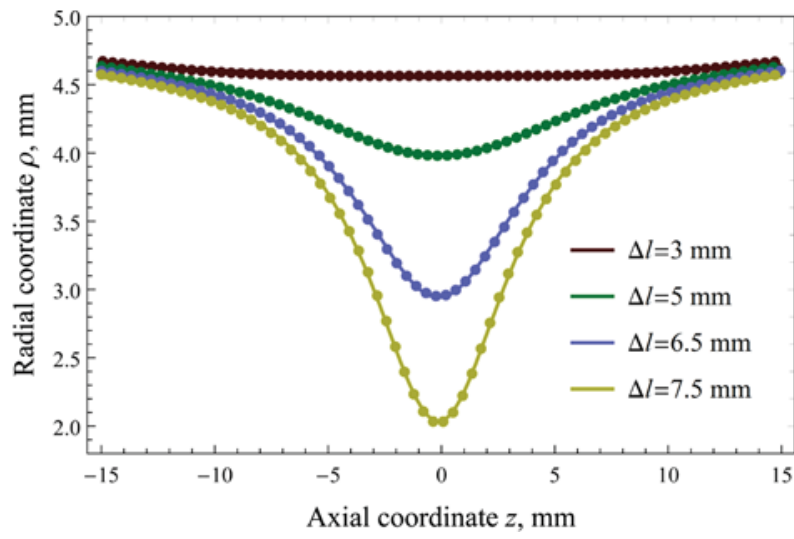

(b)

FIGURE 1. The comparison of FEM results (shown on graphs by dots) with fitted data by Eq. 1 (continuous lines): (a) neck profiles for various materials subjected to equivalent strain of about 1.5 ;

(b) neck profiles for the material with hardening curve $\sigma=600+200 \cdot \varepsilon^{0.5}$ at various instants of tension

\section{Optical Method for Measuring the Neck Profile}

There is very limited information in the literature on the use of optical systems for measuring the neck of tensile specimens. One of the earliest references to this is presented in [11]. G'Shell et al. have applied the tensile testing system with the computer-aided video extensometer and the true strain-rate controller. During testing, this system automatically processed frames of $512 \times 512$ pixels in 256-level greyscale size. In [6], Sancho et al. used the Nikon D7000 DSLR camera. For specimen profile extraction, they use an image thresholding method. In order to optimize the sharpness of images authors applied additional lightning as well as painted the specimens white. Sancho et al. improved the test procedure in [7]. They used only one light placed behind the specimen and recorded the shadow produced by the specimen.

In this work, we investigated the possibility of using the shadowgraph technique as in [7], but we faced inextricable difficulty. The problem is that the use of backlight significantly illuminates the boundaries of specimen. Therefore, the neck profile extracted from frames with the use of an image thresholding method is less than the real specimen has. At the same time, the width of the illuminated area is constant while specimen necking. This leads to a varying error which is not possible to take into account for us.

To overcome this difficulty we used green backdrop. This video technique, known as chromakey, has become extremely widespread in movie production. In our research, we used the DSLR Canon 700D camera to record the test process with a resolution of $1920 \times 1080$ pixels in RGB color space. To extract individual frames from videos with a frequency of 10 fps corresponding to the output signal of the Instron 3382 test machine, the FFmpeg opensource software was used. For the last frame, we pointed the image region of interest in which the specimen necking. The image processing procedure for measuring the neck profile involves several steps:

1. Background removal.

2. Alignment of the image in order to match the axis of specimen with the vertical.

3. Extraction of the neck profile using the Sobel filter.

4. Determination of the coordinates of points on the specimen surface in millimeters.

5. Fitting of the neck profile on both sides of specimen using Eq. 1 using the least squares method. Determination of specimen diameter in the minimum cross-section as the sum of two radii.

6. Calculation of the radius of neck curvature using Eq. 2 as the mean value of two radii.

Figure 2 shows some stages of digital image processing.

Based on the presented image-processing algorithm, we develop the program for optical measurement of the neck profile in automatic mode. 


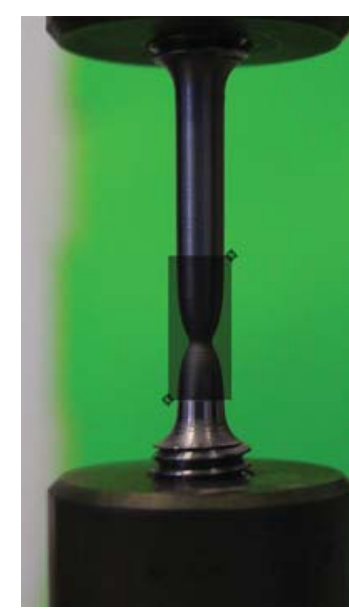

(a)

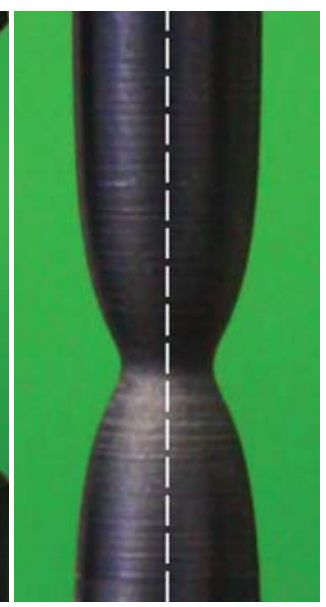

(b)

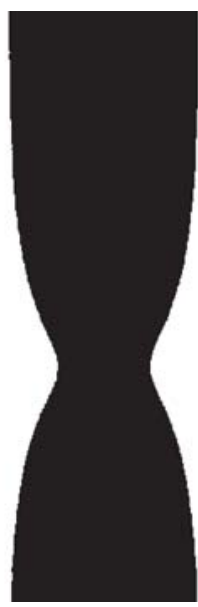

(c)

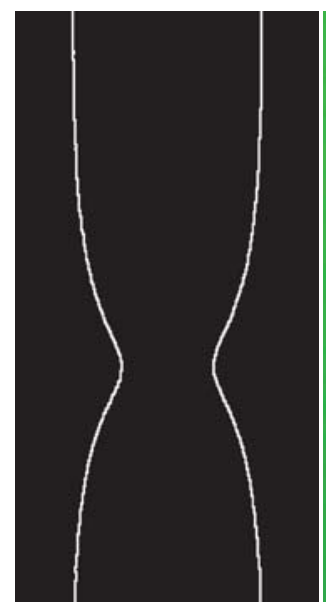

(d)

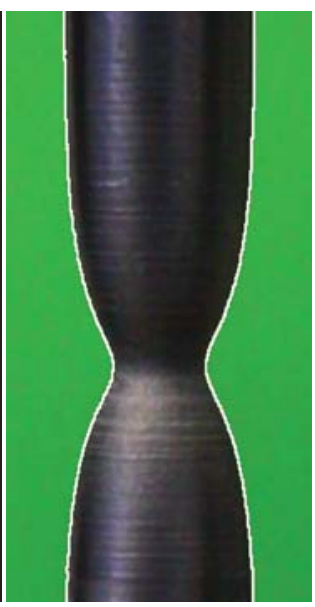

(e)

FIGURE 2. Individual stages of the image processing: (a) pointing the frame region to be processed;

(b) alignment of the cropped image in order to match the axis of specimen with the vertical;

(c) background removal and image binarization; (d) extraction of specimen borders with the use of digital image convolution procedure; (e) sample image with borders highlighted

\section{Stress and Strain Calculation}

Stresses and strains during tensile testing of specimens are most commonly represented by engineering values:

$$
\begin{gathered}
\sigma_{\mathrm{e}}=\frac{4 P}{\pi d_{0}^{2}}, \\
\varepsilon_{\mathrm{e}}=\frac{l-l_{0}}{l_{0}},
\end{gathered}
$$

where $d_{0}$ is initial specimen diameter, $l_{0}$ is original gauge length and $l$ is current value of gauge length.

However, engineering values of strain are not additive. Therefore, in this work the tensile plastic strain, also called the uniform true strain or natural strain, is often calculated as:

$$
\varepsilon_{\mathrm{p}}=\ln \frac{l}{l_{0}}
$$

Having a video record of the tensile test process, you can measure the diameter of specimen in any region of the interest at each instant of the test. This allows you to take into account the effect of the necking. However, the accuracy of measuring the diameter at the initial stage of the test is limited by the resolution of frames. In addition, it is assumed that before necking starts, the deformation is uniformly distributed throughout the gauge length. Therefore, in this work, the diameter of the specimen at the stage of uniform deformation was calculated by the formula:

$$
d=d_{0} \frac{l_{0}}{l} .
$$

After necking starts, i.e. you can see the maximum on the load diagram, the diameter of specimen in the minimum cross-section as well as the radius of neck curvature were determined on the basis of images according to the above procedure.

The average axial stresses in the neck called also the true stresses are determined by the formula: 


$$
\sigma_{\text {true }}=\frac{4 P}{\pi d^{2}}
$$

We applied the most used method to evaluate the von Mises equivalent stress from the average axial stress according to the Bridgman theory:

$$
\sigma_{\mathrm{eq}}=\sigma_{\text {true }} \cdot\left[\left(1+4 \frac{R}{d}\right) \cdot \ln \left(1+\frac{d}{4 R}\right)\right]^{-1} .
$$

Finally, equivalent strain values were calculated according to the expression:

$$
\varepsilon_{\mathrm{eq}} \approx \varepsilon_{\text {true }}=2 \ln \frac{d_{0}}{d}
$$

\section{Material and Test Setup}

The research material was GOST steel grade 09G2S melted under industrial conditions and having the following chemical composition (wt. \%): $0.08 \mathrm{C}, 1.45 \mathrm{Mn}, 0.34 \mathrm{Si}, 0.021 \mathrm{~S}, 0.018 \mathrm{P}$. For contrasting the structural and phase composition, the billets were subjected to the following treatments:

1. cold plastic deformation by rolling with overall reduction $\sim 30 \%$ (the initial state was normalized);

2. normalization at $950^{\circ} \mathrm{C}$ in order to obtain ferrite-pearlite structure;

3. quenching from $800^{\circ} \mathrm{C}$ in order to obtain ferrite-martensite structure;

4. quenching from $950^{\circ} \mathrm{C}$ and tempering at $600^{\circ} \mathrm{Cin}$ order to obtain ferrite-carbide structure.

Taking into account the aim of this research, only one specimen was prepared for each structural and phase composition of steel selected. In accordance with the type of treatment, specimens were marked: specimen-1, specimen-2, specimen-3 and specimen-4. We used standard cylindrical tension test specimens with the original gauge length equal to $25 \mathrm{~mm}$. Initial diameters were equal to 5.06, 5.04, 5.04 and $5.1 \mathrm{~mm}$ respectively.

Tensile tests were carried out on the Instron 3382 machine with a tensile speed of $2 \mathrm{~mm} / \mathrm{min}$.

The test process was recorded on a camera mounted on a tripod. While recording, overhead illumination was used with no additional lighting of specimen or backdrop.

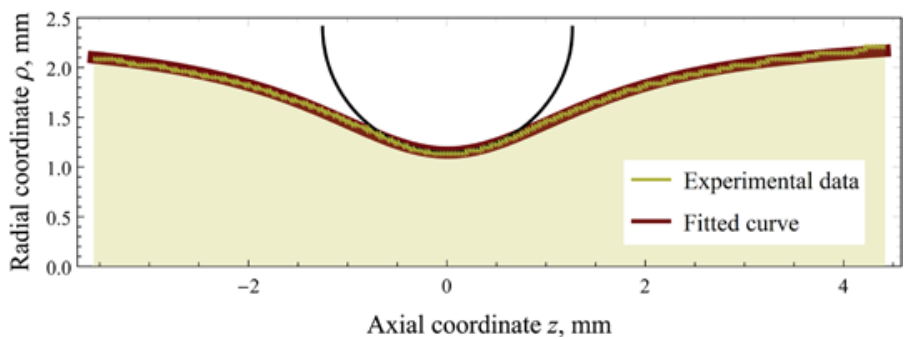

FIGURE 3. The approximation of experimental neck profile of specimen-4 immediately before fracture

\section{RESULTS}

Using the developed program, all frames corresponding to the generation and growth of the neck for each specimen were processed. The number of used frames varies with the duration of the tests. A total of 1543 images were processed, which took approximately 16 minutes of machine time on a personal computer with average technical specifications (core i7-2600 processor with 4 Gb RAM).

During the processing of each image, the accuracy of approximating the neck profile using Eq. 1 was evaluated. To assess the accuracy, we used the largest difference between the actual radial coordinate of the points on the specimen surface and the corresponding calculated value in accordance with the fit. The largest errors were 0.113 $\mathrm{mm}$ for specimen-1, $0.070 \mathrm{~mm}$ for specimen-2, $0.072 \mathrm{~mm}$ for specimen-3 and $0.066 \mathrm{~mm}$ for specimen-4. It should 
be noted that the average size of the pixel was equal to about $0.031 \mathrm{~mm}$. Figure 3 allows you to estimate the accuracy of the neck approximation.

When processing experimental data, the program calculates the values of engineering, true and equivalent stresses, as well as tensile plastic strain and equivalent strain values. Figure 4 shows the results of these calculations.

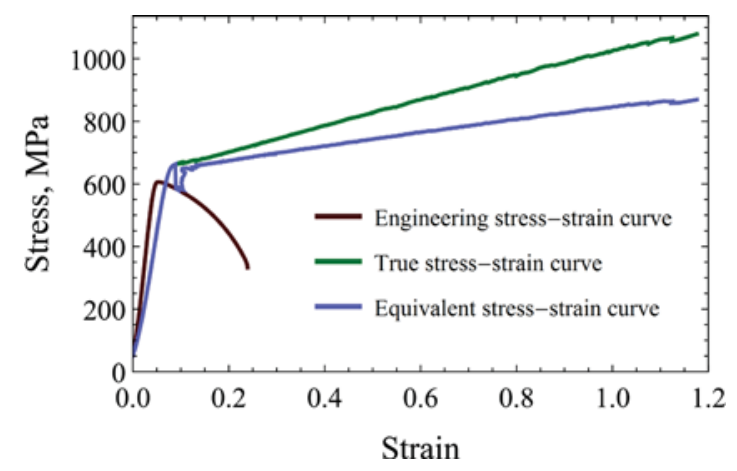

(a)

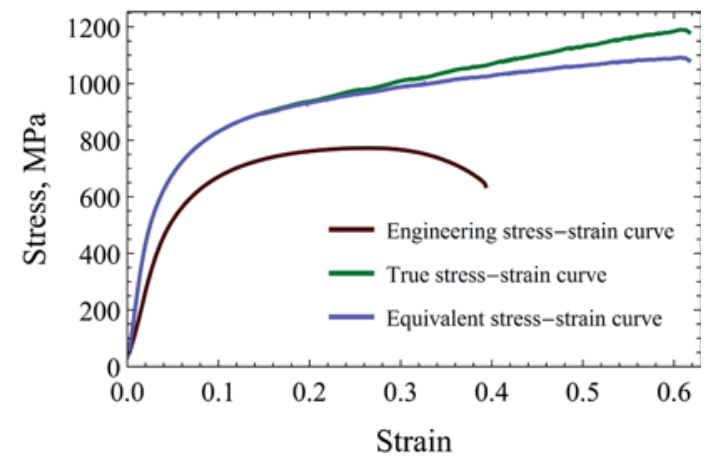

(c)

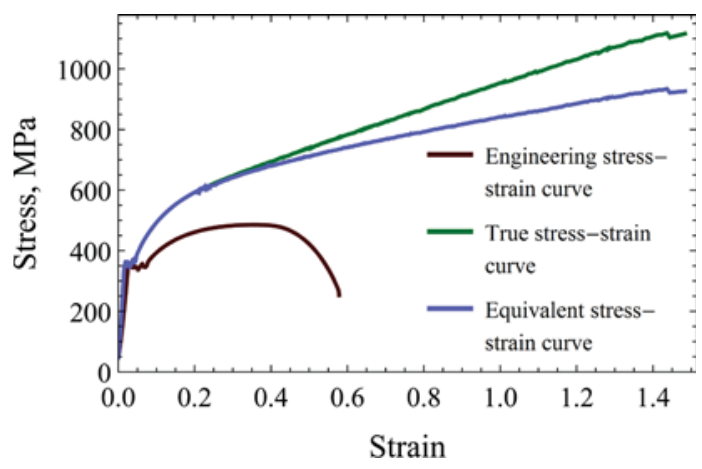

(b)

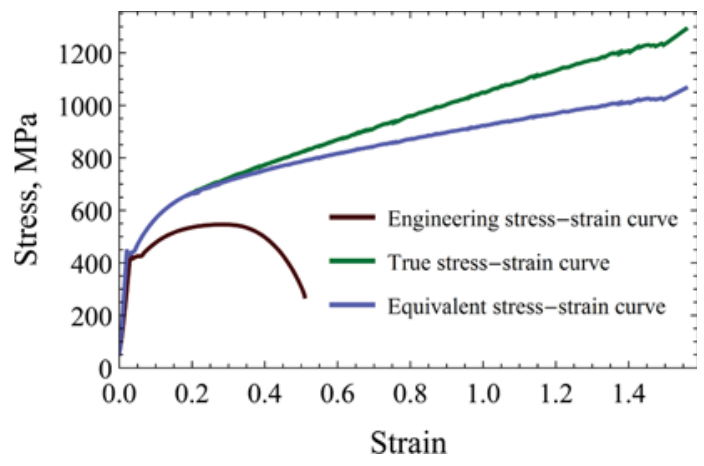

(d)

FIGURE 4. Experimental stress-strain curves for 09G2S steel: (a) specimen-1 after cold plastic deformation; (b) specimen-2 after normalization; (c) specimen-3 after quenching; (d) specimen-4 after quenching and tempering

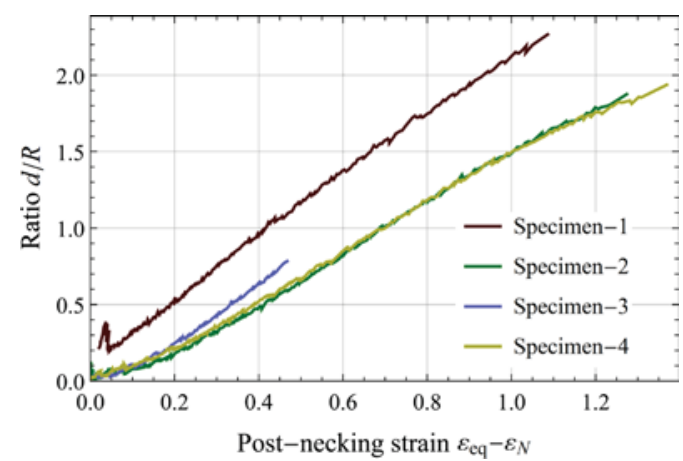

FIGURE 5. The influence of the treatment of 09G2S steel on the necking behaviour

Some authors state that a change in the neck profile occurs in the same manner regardless of the material properties $[8,9,12]$. This makes it possible to exclude experimental measurements of the neck, replacing them with statistical models to calculate the parameters reflecting the relationship of equivalent stress and average tensile stress. In this paper, the possibility of such an approach is evaluated using the example of a single steel grade, but having a markedly different character of the hardening curve. As you can see from Fig. 5, the more accurate measurement of the neck reveals the dependence of the $d / R$ ratio on material properties. 


\section{CONCLUSIONS}

The study shows that continuous experimental measurement of the neck during tensile testing of samples is necessary to study the correct material properties. The proposed neck profile equation is able to approximate the coordinates of points on the specimen surface effectively and allows calculating the radius of neck curvature. The developed program allows determine the coordinates of points on specimen surface quickly and accurately. This makes it possible to experimentally measure the neck profile at any instant of the test process on the basis of a video recording. With the use of these developments, hardening curves of 09G2S steel in various structural and phase composition were investigated.

\section{ACKNOWLEDGMENTS}

The study was made within the base part of state job in the field of scientific activity №0836-2020-0020 and was supported by the grant from the President of the Russian Federation for young scientists - candidates of sciences MK-1878.2020.8.

\section{REFERENCES}

1. P. Bridgman, Studies in Large Plastic Flow and Fracture, second ed. (Harvard University Press, Cambridge, Massachusetts, 1964).

2. N. N. Davidenkov, and N. I. Spiridonova, Proc. ASTM 46, 1147 (1946).

3. S. V. Smirnov, D. I. Vichuzhanin, and A. V. Nesterenko, PNRPU Mechanics Bulletin 3, 146 (2015).

4. D. Gerbig, A. Bower, V. Savic, and L. G. Hector,Int. J. Solids Struct. 97-98, 496 (2016).

5. J. Kajberg, K. J. Sundin, L. G. Melin, and P. Ståhle, Int. J. Plast. 20, 561 (2004).

6. A. Sancho, M. J. Cox, T. Cartwright, C. M. Davies, P. A. Hooper, and J. P. Dear, Int. J. Solids Struct. 176-177, 191 (2019).

7. A. Sancho, M. J. Cox, G. Aldrich-Smith, T. Cartwright, C. M. Davies, and P. A. Hooper, EPJ Web Conf. 183, 02063 (2018).

8. G. La Rosa, G. Mirone, and A. Risitano, Metall. Mater. Trans. A 34A, 615 (2003).

9. G. Mirone, Int. J. Solids Struct. 41, 3545 (2004).

10. M. V. Erpalov, D. Sh. Nukhov, and D. A. Pavlov, J. Chem. Technol. Metall. 55, 544 (2020).

11. C. G'Sell, J. M. Hiver, A. Dahoun, and A. Souahi, J. Mater. Sci. 27, 5031 (1992).

12. G. LeRoy, J. Embury, G. Edwards, and M. F. Ashby, Acta Metallurgica 29, 1509 (1981). 